\title{
An Application Research on Configuration Software System Platform Based on Component Technology
}

\author{
$\mathrm{Xu}$ Chao ${ }^{1} \&$ Ge Hongmei ${ }^{1}$ \\ ${ }^{1}$ Department of the Information Management, Xuzhou College of Industrial Technology, Xuzhou, China \\ Correspondence: Xu Chao, Department of the Information Management, Xuzhou College of Industrial \\ Technology, Xuzhou, China. E-mail: xuch@mail.xzcit.cn
}

Received: March 13, 2012 Accepted: April 10, 2012 Online Published: May 1, 2012

doi:10.5539/cis.v5n3p76

URL: http://dx.doi.org/10.5539/cis.v5n3p76

Fund: 2010 University research industry promotion project. NO. JHZD10-30

\begin{abstract}
This paper proposes a component based configuration software platform model that aims to develop the application system meeting different demands and build a high-quality software integration system. The model separates system logic description from system implementation and reinforces logic extensibility and reusability using the configuration idea of industrial controlling automation for reference on the base of traditional software developing method. From the effects of the application for the real projects, we can see that the method can reduce the system implementation cost and reinforce the software credibility with better maneuverability and reusability.
\end{abstract}

Keywords: component technology, configuration model, management system, software platform

\section{Introduction}

With the development of social information, the increasing scale of software system and more and more open application environment, the software application should meet the diversified demands. Software improving production efficiency and reinforcing management in the past becomes an indispensable element in people's life and work when it can help enterprises gain much more benefits. But most of time software will cause the great damage for its fault and failure (Michael \& David, 2005). How to develop the application software system meeting different demands and build up high-quality software integration system using software component is the best solution to the problem.

Software component technology supporting applied development environment and system through component integration includes component language, component category, component extraction, component model, component assembly and component credibility etc. Now, there are a lot of mature component technologies that are emphasized by many large enterprises and software component technology has become the main method to improve software quality and solve software crisis. The development of software system implemented by assembly and integration of the existing components can reduce software development cost, shorten development period and enhance the whole quality of software system (Fan, Li, \& Jiang, 2011). Therefore, the research on software component technology is important.

Computer room management system mentioned in this paper utilizes the updated research findings of software component technology, draws lessons from configuration idea of industrial controlling automation on the base of the method of traditional software development and gains reliable configuration tool software by building engineering principle. This helps software face the end-users with dynamic demands, enhance the speed and efficiency of the software development by dynamically integrating reusable component and equipping personalized application system, improve the flexibility and reconfiguration of application system and make a beneficial research on automation and industrialization for software production.

In addition, this paper is closely combined with the real application program New-Generation Application platform of Monitoring Configuration Software for Computer Room, the industrial promoting program as achievement in scientific research of university in Jiangsu. 


\section{Configuration Model for Component Software System}

This paper puts forward a configuration model shown in Figure 1 for component software system which can separate system logic description from system implementation. The model is composed of configuration virtual computer, system structure, and component and system logic description. In this model the software component is the object implementing specific logic function in system and the application software is the loose coupling reconfiguration system that the components are connected through macro logic configuration description. The model can reinforce logic extensibility, flexibility and reconfiguration of software system for application demands.

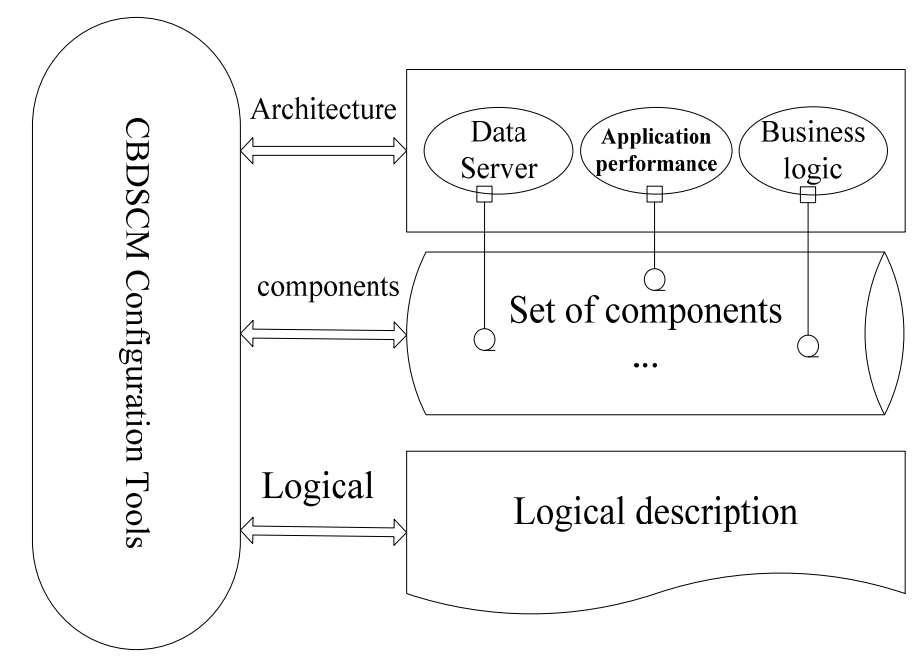

Figure 1. Component based dynamic software configuration model

\subsection{Component Model}

The component model follows the principle separating the definition of interfaces from implementation with the reference to UDM digital model (Reuse Library Interoperbaility Group, 1994). The implementation of component will be designed with the reference to the component implementation model such as DCOM and Java Beans that can be encapsulated with related description information of components. Representation of component will be described with the knowledge representation of framework of artificial intelligence (Frakes \& Nejmeh, 1990; Mihi et al., 1994; Chen et al., 1993). The property of component will be divided into natural property groove, correlative property groove and property of interfaces method with framework's groove. The specific knowledge representation is shown in Figure 2.

Each component will be described with a framework knowledge representation and property will be described with groove. The groove with many sides represents the content of property. Each side with many values describes the different property value of component. 


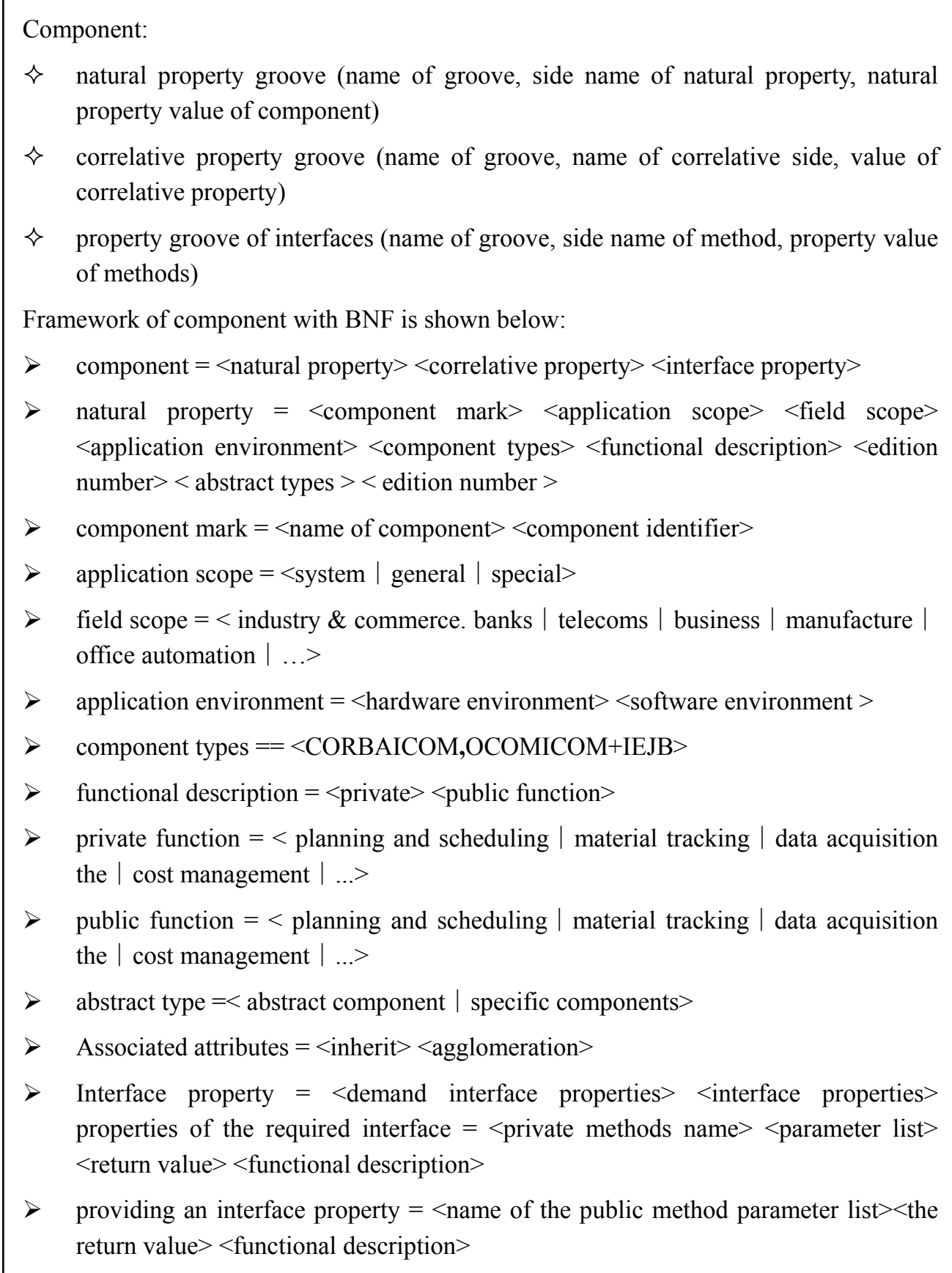

Figure 2. Component model

\subsection{System Structure}

System structure of software is described by software architecture style which is a kind of software system structure. Each architecture style represents a group of system structure of software with similar property. Architecture style defines a software system family by a vocabulary containing some components and connectors' types and a group of constraints showing how to connect the components with connectors (Brownsword \& Clements, 1996; Sackett \& Maxwell, 1997). Distributed architecture adopted in configuration model is shown in Figure 3. 


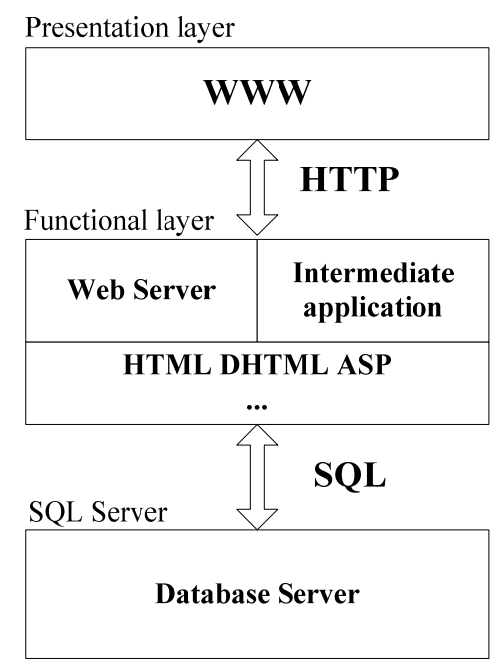

Figure 3. The architecture of web-based distributed system

The feature of the model is that the client can access the database by severs and an intermediate application program is designed as a client agent to severs. Actually the intermediate application program plays a role of conversion between two different application, HTML file access and database access. When a technology of intermediate application program is chosen, a group of standard parameter formats and communication protocol will be defined. Communication interfaces between intermediate application program and database follow SQL standards and are implemented by the technology of ODBC, ADO and JDBC.

\subsection{Configuration Logics}

Configuration logics refers to the abstraction for software system with $\mathrm{CD}=(\mathrm{Cs}, \mathrm{E}, \mathrm{p}, \mathrm{Ct}, \mathrm{B})$ of system configuration description.

Configuration logic mainly refers to the five tuples $\mathrm{CD}=(\mathrm{Cs}, \mathrm{E}, \mathrm{p}, \mathrm{Ct}, \mathrm{B})$ that described by system configuration. It abstracts software system, identifies the component, component interface and system action in the system uniquely, describes the rules of the state change abstractly in the system control sets, and maps every action sequence of events into the system the next action (Anderson, 1997; CMM I Product Team, 2000). It can ensure the effective operation of application systems, and implementing the interactive link between the components through the component interface.

Control statement is the basic unit which controls the actions of software system. The definition is as follows:

Action $p=<$ control statements $>$

control statements $=<$ name of component $><$ component inlet $><$ component outlet $\rangle<$ constrains $>$

Each control statement can guide the software system of configuration to cite the next appointed component in specific way.

The execution result of control statement will monitor the next message send by component example. The messages generated by all control statements in each control interface will form message sequence $\mathrm{X}$ which is the judgment basis of dominating set of system.

\section{Design and Implementation of Configuration Platform for Computer Room Management System}

Configuration model for component software system can solve the problems of maintainability, expansibility, reconfiguration and flexibility existing in the traditional application system. The implementation of model relies on a configuration platform which can provide a simple but visual operation interface to implement a large number of operation logics of system and subject directly for the users with dynamic demands. It can help the users who know the management of operation logics without the knowledge of software development directly uses the tool software by the means of the configuration of operation components. Taking New-Generation Application platform of Monitoring Configuration Software for Computer Room as an example, this paper demonstrate how to operate. 


\subsection{Design of Configuration Platform}

On the base of configuration model of component software system, configuration platform for computer room not only provides the visual and convenient configuration operation interface but also contains a large amount of field components managing and maintaining the components of software such as adding, browsing, searching and deleting. The platform can display and record effectively the operation courses and results of configuration. For the customization results of configuration the platform can convert to application system. Framework of platform is shown in Figure 4 including five modules:

- Main Framework: It is the micro-core of the platform in charge of loading, configuration, unloading, starting, stopping and suspending in other function modules.

- Reusable Component System: It is the premise and base of configuration providing the sets of function components for configuration operation.

- Visual Configuration Interface: It can provide visual and convenient operation interface for the end-users.

- Program Management: It can record the operation course and results of configuration with the support of engineering file, page file, configuration file, intermediate file of database configuration by the means of the category of engineering tree. The operation results of configuration are the operation rules and logic process of application software system based on the configuration model of reusable components technology and the research of platform.

- Uploading Program: It can not only upload the projects but also deploy and convert application system according to the record results of configuration.

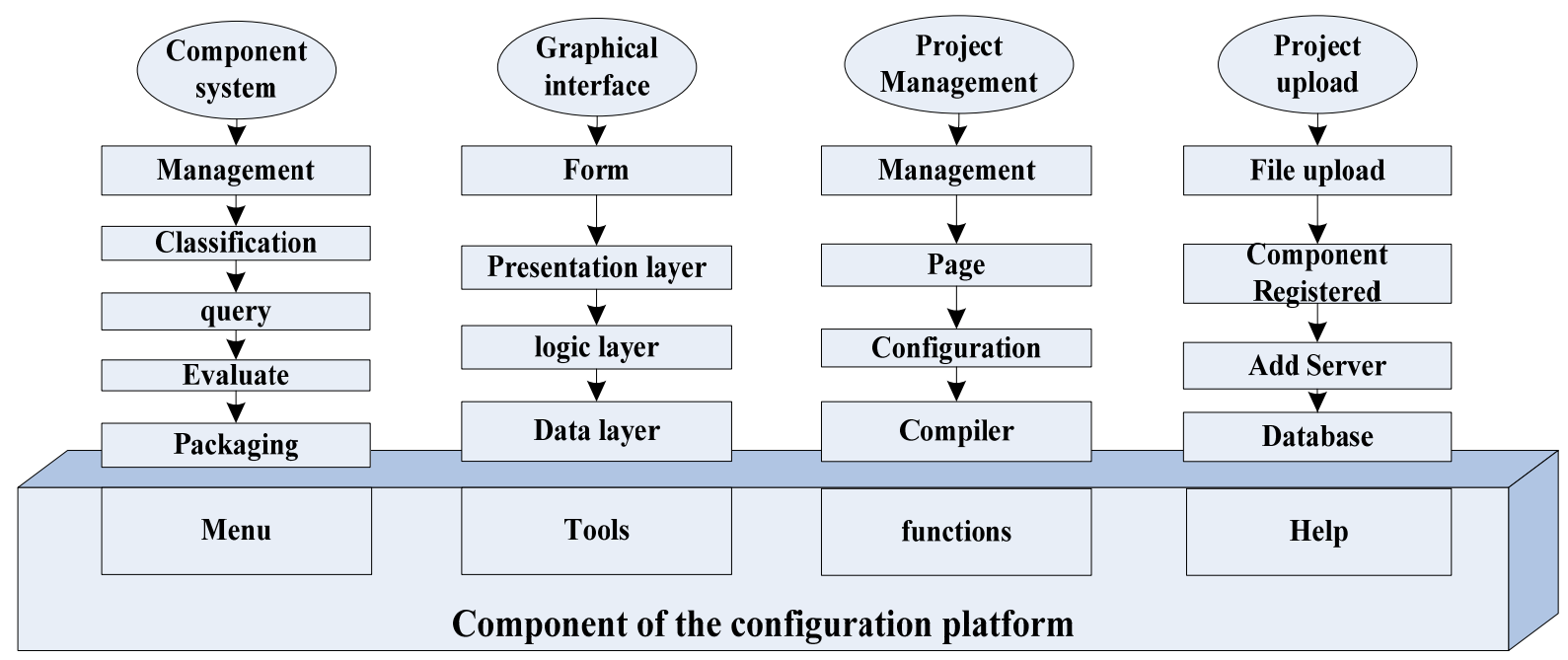

Figure 4. Framework of configuration platform of information system

\subsection{Implementation of Configuration Platform}

The configuration management system for computer room implements the platform system of configuration integrating in the development environment of visual studio 2010 based on the technology of components. The module of database generation, the module of system compile and the module of file transmission in the backstage implement the final application system. The platform of configuration implements the visual operation interface like Windows style. It includes 7 systems such as distribution monitor, air condition monitor, temperature and humidity monitor, water leakage monitor, fire protection monitor, guard monitor and video monitor shown in Figure 5. 


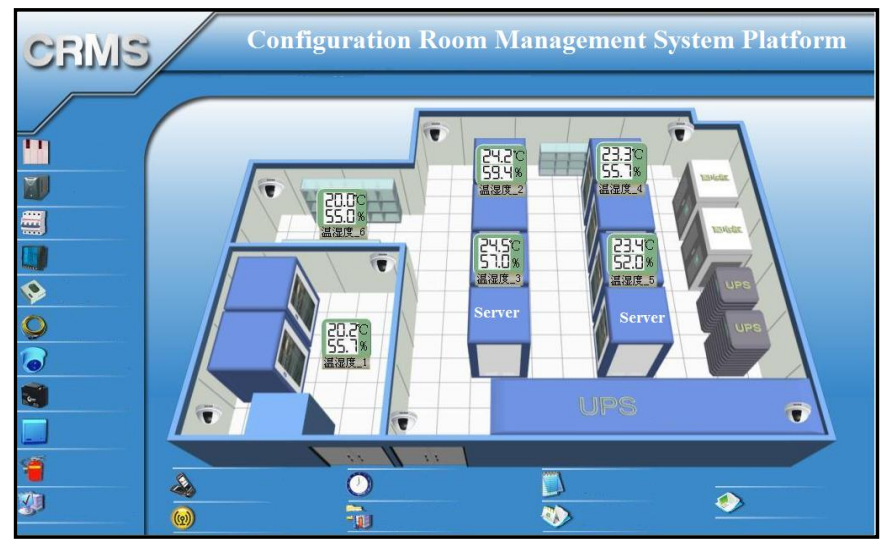

Figure 5. Main system interface

The system with open structure can support all kinds of transmission net including Ethernet, frame relay net, FDDI net, ATM net, PPP net, Token Ring etc. The hardware structure of system is shown in Figure 6.

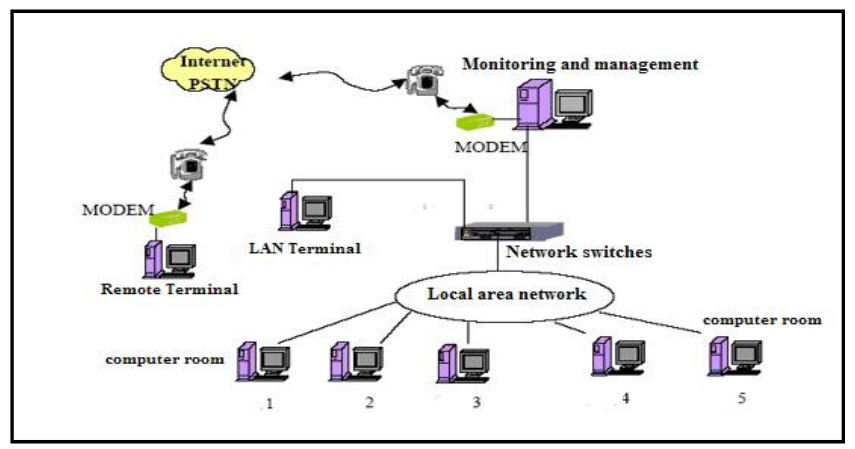

Figure 6. Hardware architecture platform

As a united and convenient information exchange platform, the real-time running information of sub-system can be uploading to the monitor management station with the system based on TCP/IP Protocol. The administrators can browse the real-time information, monitor and manage the real-time working condition through monitor terminal with the authority. The comprehensive information and data will be provided by ODBC technology connecting SQL database with management information database.

Desktop users can access and control lower monitor sub-system through DCOM when DCOM is used in the system. The structure of software system is shown in Figure 7.

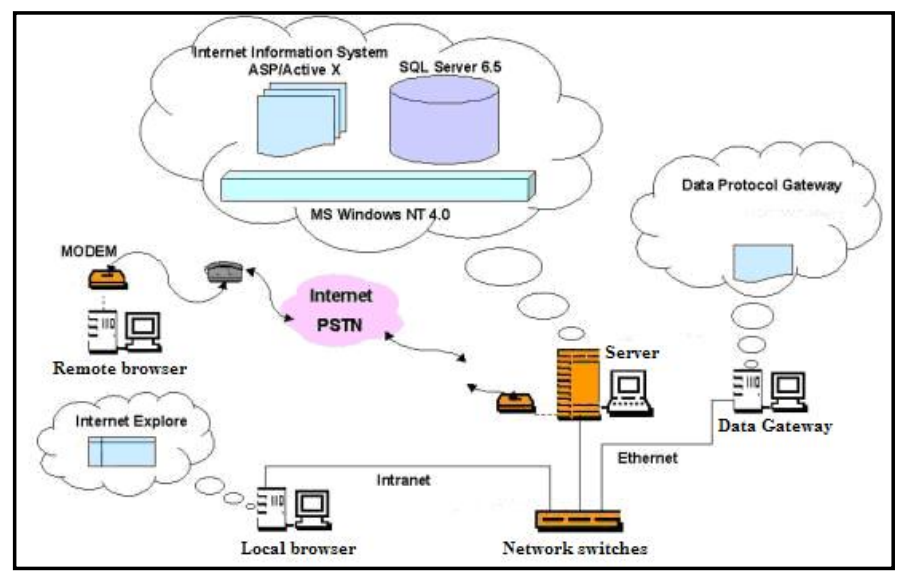

Figure 7. Software architecture platform 
At present information management system of customized implementation for configuration platform has been applied in many schools in Jiangsu with good feedback. For the design of reusable software components and the development of configuration platform of information system is relatively slow, the rapid customization could meet the markets' demands better.

From the real application example of this method, we can see that it will be useful and practical.

\section{Conclusion}

By analyzing technology of software components both at home and abroad, this paper concludes the main features of present component technology and deficiency in specific application field. With the real application program of New-Generation Application platform of Monitoring Configuration Software for Computer Room, this paper puts forward a configuration model of component software system to solve the visual assembly of components and designs the configuration platform of information system to implement prototype system providing the reliable assembly supporting platform for configuration platform of reusable components. This paper develops a real example model with shortening product development period, the end-users oriented, high coherence between software application and demands, large development scale and low development cost on the base of configuration customization. All the analysis shows that it is meaningful for rapidly developing a high-quality software system.

\section{References}

Anderson, D. M. (1997). Agile product development for mass customization. New York: Mcgraw-hill.

Brownsword, L., \& Clements, P. (1996). A case study in successful product line development. Software Engineering Institute, Tech Rep: CMU/SEI-96-TR-016.

Chen, P. S., et al. (1993) On the retrieval of reusable component. In: the 2nd Int workshop on software reusability, Advances in Software reuse. Lucca, Italy.

CMM I Product Team. (2000). CMM I for systems engineering/software engineering/integrated product and process development. Software Engineering Institute, Tech Rep: CMU/SEI-2000-TR-031.

Fan, D., Li, Z., \& Jiang, X. (2011). Code optimization and program criterion conversion in certifying compiler [J]. Micro computer system, 7, 1400-1405.

Frakes, W. B., \& Nejmeh, B. A. (1990). An information system of software reuse. In: Software reuse: Emerging technology. IEEECS Press, pp. 142-151.

Michael, H., \& David, L. (2005). Writing secure code. Redmond, Washington, USA: Microsoft Press.

Mihi, H., et al. (1994). Intelligent component retrieval for software reuse. In: Proc. 3rd Maghrebian Conefernce on Al and SE, Rabat, Morocco, pp. 101-114.

Reuse Library Interoperbaility Group. (1994). Uniform data model for reuse libraries (UDM). RIG: Technical Report: 0002.

Sackett, P. J., \& Maxwell, D. J. (1997). Customizing manufacturing strategy. Integrated Manufacturing System, 8(6), 359-364. http://dx.doi.org/10.1108/09576069710188779 